Imploded Capsule Fuel Temperature and Density Measurement by

Energy-Dependent Neutron Imaging

M. J. Moran, J. Koch, O. L. Landen, S. W. Haan, C. A. Barrera, E. C. Morse

October 12, 2005

2005 Fourth International Conference on Inertial Fusion Sciences and Applications

Biarritz, France

September 4, 2005 through September 9, 2005 
This document was prepared as an account of work sponsored by an agency of the United States Government. Neither the United States Government nor the University of California nor any of their employees, makes any warranty, express or implied, or assumes any legal liability or responsibility for the accuracy, completeness, or usefulness of any information, apparatus, product, or process disclosed, or represents that its use would not infringe privately owned rights. Reference herein to any specific commercial product, process, or service by trade name, trademark, manufacturer, or otherwise, does not necessarily constitute or imply its endorsement, recommendation, or favoring by the United States Government or the University of California. The views and opinions of authors expressed herein do not necessarily state or reflect those of the United States Government or the University of California, and shall not be used for advertising or product endorsement purposes. 
The headers will be insert by the Publisher

The headers will be insert by the Publisher

The headers will be insert by the Publisher

\title{
Imploded Capsule Fuel Temperature and Density Measurement by Energy-Dependent Neutron Imaging
}

\author{
Michael J. Moran* and Jeffrey Koch* and Otto L. Landen* and Steven W. \\ Haan* $^{*}$ and Carlos A. Barrera** and Edward C. Morse**
}

*LLNL, P.O. Box 808, L-481, Livermore, CA 94550 USA

** University of California, Dept.of Nuclear Engineering,Berkeley, CA 94720 USA

\begin{abstract}
Neutron imaging systems measure the spatial distribution of neutron emission from burning inertial confinement fusion (ICF) targets. These systems use a traditional pinhole geometry to project an image of the source onto a two-dimensional scintillator array, and a CCD records the resulting scintillation image. The recent history of ICF neutron images has produced images with qualities that have improved as the fusion neutron yields have increased to nearly $10^{14}$ neutrons. Anticipated future neutron yields in excess of $10^{16}$ at the National Ignition Facility and LMJ have raised the prospect of neuron imaging diagnostics which simultaneously probe several different characteristics of burning fusion targets. The new measurements rely on gated-image recording to select images corresponding to specific bands of neutron energies. Gated images of downscattered neutrons with energies from 5 to $8 \mathrm{MeV}$ can emphasize regions of the target which contain DT fuel which is not burning. At the same time, gated images which select different portions of the $14-\mathrm{MeV}$ spectral peak can produce spatial temperature maps of a burning target. Since the neutron production depends on the DT fuel density and temperature, simultaneous images of temperature and neutron emission can be combined to infer the an image of the source density using an Abel inversion method that is analogous to the method that has been used in x-ray imaging. Thus, with higher-yield sources, neutron imaging offers the potential to record simultaneously several critical features that characterize the performance of an ICF target: the neutron emission distribution, the temperature and density distributions, and the distribution of nonburning fuel within the target.
\end{abstract}

Neutron-based images of burning inertial confinement fusion (ICF) targets can be used to study several different aspects of fusion physics. The traditional application is to use an image which is integrated over the entire duration of the fusion burn to provide a 2-dimensional map of the spatial distribution of DT fusion burn. The 2-dimensional display distorts the apparent shape of the source because each image pixel is, in fact, an integral through the target at that position, as illustrated in Figure 1. The integral results from the high transparency of the compressed target to the neutrons that are produced by DT fusion.

As neutron imaging techniques become more sophisticated, the images can be recorded in ways that reveal the internal physics of the fusion burn. Previous publications have shown that gated images recorded at neutron flight times that correspond to downscattered neutron energies from 6 to $10 \mathrm{MeV}$ 

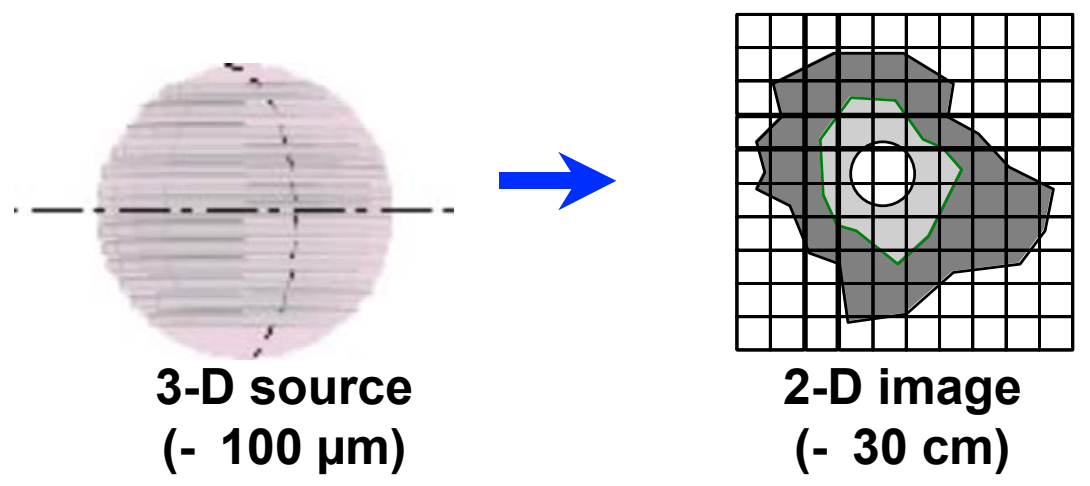

Figure 1. 3-D source projected onto 2-D image.

can reveal the spatial distribution of nonburning fuel that surrounds the hot burning core of the target (see Figure 2$)^{1}$. These measurements are possible because the optical emissions from modern fast scintillators decay quickly enough that low-intensity late-arriving downscattered images can be recorded after the initial $14-\mathrm{MeV}$ peak.

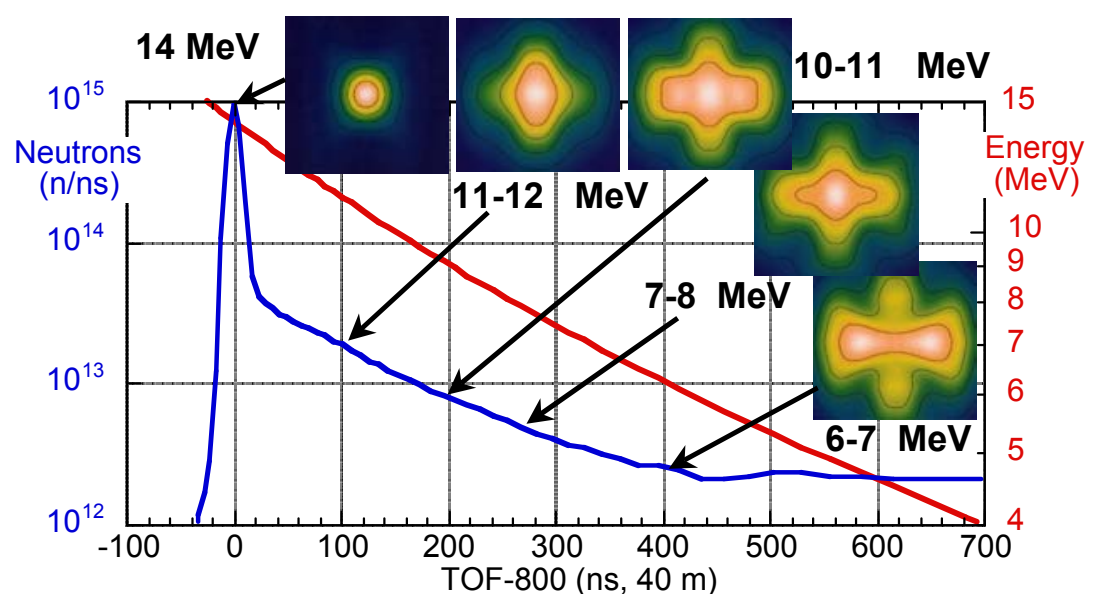

Figure 2. Neutron images versus neutron energy.

Another application that has been considered is fast gating of multiple $14-\mathrm{MeV}$ neutron images. The relative intensities of these images depend on the temperature-dependent spectral width of the 14$\mathrm{MeV}$ peak. A pixel-by-pixel map of the ratios of images at different energies near $14-\mathrm{MeV}$ provides a direct indication of the temperature distribution over the hot burning core. ${ }^{2}$ With a sufficiently long line of sight $(\approx 40 \mathrm{~m})$ and fast scintillators, the recorded ratios provide a good indication of the 14$\mathrm{MeV}$ spectral width, as illustrated in Fig. 3. Here, burn temperatures of $10 \mathrm{keV}$ and $50 \mathrm{keV}$ lead to spectral widths of $560 \mathrm{keV}$ and $1260 \mathrm{keV}$, respectively, for the $14-\mathrm{MeV}$ peak. Gated images at different times on the peak will have ratios that reflect the underlying spectral widths.

The independent images of emission intensity and burn temperature make it possible to study the density distribution of a burning ICF target. This is because neutron emission $\mathrm{dn} / \mathrm{dt}$ can be written simply as $\mathrm{dn} / \mathrm{dt}=\mathrm{N}^{2} \operatorname{sv}(\mathrm{T})$, where $\mathrm{N}$ is the (equimolar) deuterium and tritium density and $\mathrm{sv}$ is the known temperature-dependent Maxwell-averaged DT fusion cross section. With measurements of the emission distribution ( $\mathrm{dn} / \mathrm{dt}$ ) and temperature distribution $(\mathrm{T})$, the equation can be inverted to provide 


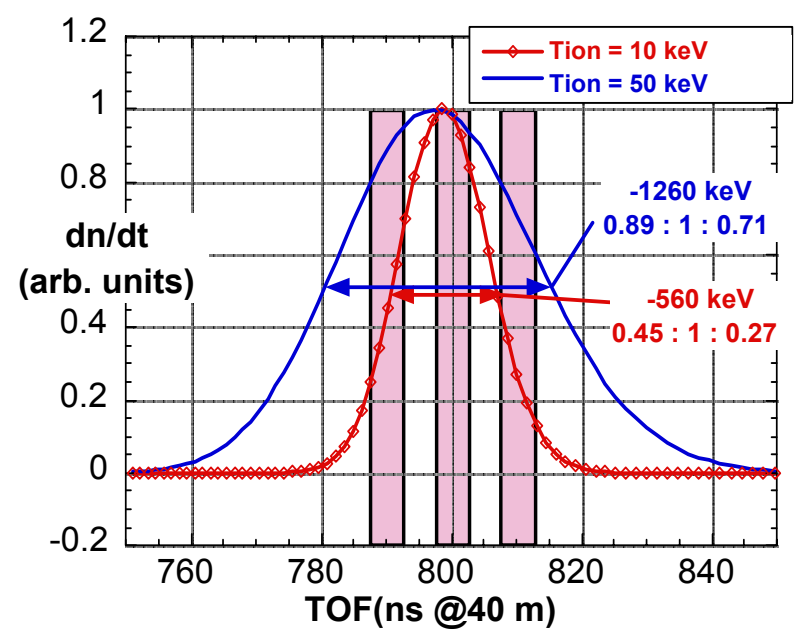

Figure 3. Variation of image intensity versus gate window time(pink).

the spatial distribution of the DT density: $\mathrm{N}=((\mathrm{dn} / \mathrm{dt}) / \mathrm{sv}(\mathrm{T}))^{1 / 2}$, where the position dependences of $\mathrm{N}$, $\mathrm{n}$, and $\mathrm{T}$ have been suppressed, and where the density is described, pixel by pixel, by a columnaveraged value through the target.

Further analysis of the data can characterize the full three-dimensional density distribution $\mathrm{n}(\mathrm{x}, \mathrm{y}, \mathrm{z})$ in the target. This analysis is directly analogous to the "Abel inversion" method that has been used with $x$-ray imaging infer the density distribution of compressed ICF targets. ${ }^{3}$ The x-ray images use the independent intensity and temperature measurements, combined with key assumptions regarding the symmetry of the target shape, to derive the three-dimensional density profile. Similarly, with neutron imaging, the neutron emission image and temperature map can be used to characterize the spatial density profile when key assumptions regarding the target symmetry are allowed.

In conclusion, neutron imaging experiments have the potential to provide much more than a simple image of a burning ICF target. Gated images can provide the distribution of nonburning fuel outside of the hot core, and they also can be combined form a map of the temperature distribution across the hot core. The neutron emission image and temperature map can be combined to characterize the three-dimensional density distribution in the target cores. This work was performed under the auspices of the U.S. Department of Energy by the University of California, Lawrence Livermore National Laboratory under contract No. W-7405-Eng-48.

\section{References}

[1] M.J. Moran, S.W. Haan, S.P. Hatchett, N. Izumi, J.A. Koch, R.A. Lerche, and T.W. Phillips, Rev. Sci. Inst. 74, p. 1701 (2003).

[2] D.C. Wilson, C.R. Christensen, G.L. Morgan, M.D. Wilke, P.A. Bradley, and P.L. Gobby, Rev. Sci. Inst. 74, p. 1705 (2003).

[3] J.A. Koch, S.W. Haan, R.C. Mancini, J. Quantit. Spectros. \& Rad. Trans. 88, p. 433 (2004). 